\title{
Students' Perception of Cognitive Load in an Accelerated DPT Program with a Blended Curriculum
}

\author{
Rossniel Marinas ${ }^{1}$, Shannon Groff ${ }^{2}$, Sunddip Panesar-Aguilar ${ }^{1} \&$ Tatiana Godoy Bobbio ${ }^{1}$ \\ ${ }^{1}$ College of Health Sciences, University of St. Augustine, Florida, United States \\ ${ }^{2}$ Department of Education and Human Services, Florida State College at Jacksonville, Jacksonville, Florida, \\ United States \\ Correspondence: Sunddip Panesar-Aguilar, College of Health Sciences, University of St. Augustine, Florida, \\ 32246, United States. Tel: 1-904-770-3676. E-mail: saguilar@usa.edu
}

Received: November 9, 2021 Accepted: December 23, 2021 Online Published: January 7, 2022

doi:10.5539/gjhs.v14n2p52 URL: https://doi.org/10.5539/gjhs.v14n2p52

\begin{abstract}
Administrators and educators in higher education are interested in how academic tutoring services and gender impact perceptions of cognitive load and, therefore, students' academic success. However, a lack of evidence existed in the literature regarding physical therapy students' perception of cognitive load in an accelerated Doctor of Physical Therapy program with blended learning. Participants in this quantitative, non-experimental study completed the adapted Cognitive Load Scale to indicate their perception of cognitive load, participation in academic tutoring services, gender, and age. The DPT students perceived high cognitive overload, but a $t$ value of 0.37 and a $p$ value of 0.71 indicated that their perception was not significantly related to gender. Further, a $t$ value of -3.09 and a $p$ value of 0.005 indicated that academic tutoring services played a vital role in minimizing the perception of cognitive overload. However, the $p$ value of 0.11 of the parametric multiple linear regression analysis and the $p$ value of 0.59 of the interaction term indicated no moderating relationship between academic tutoring services and gender. This evidence may assist physical therapy administrators and educators of DPT students in re-structuring blended learning programs and accelerated curricula to reduce student perceptions of cognitive overload.
\end{abstract}

Keywords: higher education, academic success, cognitive load, physical therapy, physical therapy program.

\section{Introduction}

\subsection{Introduce the Problem}

The problem is there is a lack of evidence of research identifying the DPT students' perception of cognitive load in an accelerated PT program with a blended curriculum. No research was found examining how academic tutoring services and gender influence the perception of cognitive load in an accelerated PT program with a blended curriculum. Accelerated and rigorous graduate curricula contain high course content and coursework (Khan, 2015). No educational model is one-size-fits-all approach and not all students exhibit the same learning styles or demonstrate a clear understanding of expectations of a blended curriculum. Some students may experience cognitive overload and unsatisfactory academic performance when learning in a blended curriculum. Variables such as students' perception of cognitive load, academic tutoring services and gender need to be evaluated to facilitate learning and academic success, enhance a student-centered approach in the classroom and facilitate student well mental being (Castro-Alonso et al., 2019).

High levels of course content and coursework may lead to information overload, causing stress among graduate students and negatively influencing learning experiences and academic performance (Zotova, 2015). The learner's cognitive load needs to be at a minimum for effective permanent learning (Sweller, 2019). Since learning is most effective under conditions aligned with human cognitive architecture known as schemes, instructional designs should reduce working memory load to facilitate the changes in long term memory associated with schema acquisition (Jonker et al., 2020; Leppink \& Duvivier, 2016; Mayer, 2017). The exploration of the DPT students' perception of cognitive load on academic performance in an accelerated program with a blended curriculum can influence course and curriculum designs, enhance learning experiences, academic success, and student mental being. 
The CLT served as the framework for this study. Sweller (1988) postulated the CLT by analyzing cognitive load effects on learning during problem-solving activities. The CLT provides an understanding of the different processes of learning related to working memory and the conceptual parts of cognitive load (Sweller, 1988; Sweller et al., 2019; Wei, 2017; Zotova, 2015). Physical therapist educators need to be aware of the challenges that accelerated programs with blended learning pose on DPT students and identify the relationship between academic tutoring services and gender and the perception of cognitive load to help students develop the skills to learn synchronously and asynchronously.

\subsection{Explore Importance of the Problem}

In recent decades, the higher education landscape has shifted from traditional to blended learning due to a shortage of educators and the worldwide demand for individuals with graduate degrees and competitive skills. Online learning is attractive to students since it allows them to attend school remotely and manage their personal and work-related responsibilities. In 2015, the Lumina Foundation stablished a goal for $60 \%$ of Americans to earn a certificate, college degree, or higher-quality postsecondary credentials by 2025 . The high demands for individuals with graduate education contributed to accelerated, hybrid, and blended curricula (Picardo, 2017).

The education requirements for many healthcare professionals have also changed. The professional physical therapist education transformed, moving from a terminal master's degree to an entry doctoral degree. Many entry-level traditional and accelerated programs offer hybrid and blended curricula. To address the demand, many institutions have implemented blended learning models to replace the traditional instructional methods (Gagnon et al., 2020).

Research in pharmacy, nursing, and medical education has investigated how cognitive overload impacts students' academic performance, motivation, and mental well-being. In CLT, teaching and learning consider the students' mental effort in the working memory when learning a particular task (Leppink \& Duvivier, 2016). Leppink and Duvivier (2016) identified task fidelity, task complexity, and instructional support as core issues contributing to cognitive overload in medical programs. Task fidelity refers to initial learning from textbooks, evidence-based practice, and advanced learning from simulated patients to real patients, avoiding cognitive and emotional overload. In task complexity, the task's duration, the familiarity with the task, and the amount of information associated with the task are considered when facilitating learning from simple to more complex tasks while avoiding cognitive overload.

To further minimize the risk of cognitive overload, instructional support, such as technological, teaching, and learning resources, are gradually faded as the learner transitions from a guided step-by-step process of on how to solve a problem through completion tasks to tasks performed autonomously (Leppink \& Duvivier, 2016). Leppink and van den Heuvel (2015) designed a holistic model for integrating a competency into a medical curriculum, facilitating the transition from high instructional support on low-complexity low-fidelity learning tasks to highcomplexity tasks in high-fidelity environments carried out autonomously.

Academic tutoring is a standard supplemental service for students in higher education. Academic tutoring services provide guidance and strategies by targeting knowledge gaps and weaknesses and strengthening acquired knowledge to help learners succeed academically. Arco-Tirado et al. (2019) examined the impact of a peer-tutoring program on first-year students' academic performance by implementing the Peer-Tutoring Program version 3.0 (PTP 3.0). The PTP 3.0 main objectives were to provide strategic approaches to improve students' level of self-regulated learning to improve academic performance, facilitate social adjustment to the university study demands, and increase retention. This study's findings support using academic tutoring services as a moderator variable when examining students' perception of cognitive overload and academic performance in a blended curriculum.

Considering the CLT, the findings of the study contribute to the gap in the literature examining DPT students' perception of cognitive load in a blended curriculum, and to other healthcare programs that deliver content through a blended learning method. This research study identified the relationship between cognitive load, academic tutoring services and gender among DPT students in an accelerated program with a blended curriculum. The findings of this study can encourage the reassessment of course designs and instructional methods to minimize cognitive overload and target male and female DPT students effectively. Lastly, this study can promote the implementation of academic tutoring services to enhance academic performance.

\subsection{Describe Relevant Scholarship}

\subsubsection{Cognitive Load Theory}

Cognitive Load Theory (CLT) is a framework that provides an understanding of the different processes of learning 
related to working memory and the conceptual parts of cognitive load (Sweller, 1988). Cognitive load is the limited capacity of information the working memory can store. CLT is built on the foundation established by other constructs such as working and long-term memory and the correlation to capacity. The interplay between sensory components such as vision and auditory combined with cognitive capacity may explain learning variability (Mayer, 2017). The ability to process information may be determined by how it is organized, indicating that instruction has a significant impact while utilizing extensive cognitive processing can impact the ability to acquire new knowledge (Sweller et al., 2019; Sweller, 1988).

\subsubsection{Interplay Between Aspects of Cognitive Load}

Consensus regarding the measurement of the various forms of cognitive load is difficult due to challenges identifying and differentiating the three forms of cognitive load. Researchers may choose to use various study designs, including experimental, quasi-experimental, and exploratory, when examining cognitive load. The studies selected in this literature review focused primarily on college students who volunteered to participate as subjects, and most were a convenience sample, and, in some cases, they were compensated. Some studies have smaller samples, while others had over a thousand participants. When assessing cognitive load, information may be presented online utilizing various multimedia formats with fewer face-to-face encounters. Studies may choose to use subjective or objective measures of cognitive loads with some studies validating the tools during the studies. Good correlation exists between objective and subjective cognitive load measures (Nazari et al., 2020).

Klepsch et al. (2017) found that the participants reported more demands on all types of cognitive load compared to low load activities when comparing high load tasks to ICL, ECL, and GCL. The Klepsch et al. (2017) results were confirmed by Nazari et al. (2020). In the study conducted by Nazari et al. (2020), the students participated in an introductory surgical course and identified increased GCL/ICL compared to decreased ECL based on instruction variation. Costley and Lange (2017) examined the diversity of content and the impact on GCL. The research findings confirmed that a higher germane load was related to multimedia learning content diversity and increased student comprehension.

Overall, the research appears to support the relationship between the three aspects of the CLT (Costley \& Lange, 2017; Nazari et al., 2020). The research consistently indicated that improved knowledge and skill acquisition occurs when germane cognitive load is high relative to the extraneous cognitive load. While students generally performed better when cognitive load is managed, the research did not examine specific trends related to the three aspects of cognitive load and student GPA. Most of the studies did not determine the students' perception and gender related trends, indicating that it's an area that requires additional research.

\subsubsection{Load Reduction Instruction Scale}

Educational environments comprise students with a diverse range of skills and knowledge. Instructional approaches must accommodate the different skills and knowledge sets in the classroom to facilitate learning more effectively. As the learner improves his skills and enhances his knowledge, instructional designs should facilitate autonomous learning. The architecture of the human mind and its memory systems are the fundamental principles underpinning the rationale for the Load Reduction Instruction (Martin \& Evans, 2019). Martin and Evans (2019) investigated the validity of the Load Reduction Instruction Scale (LRIS) and its underlying conceptual contentions. The sample consisted of 393 high school students from 40 mathematics classrooms in two independent schools in Sydney, Australia. Martin and Evans (2019) concluded that the LRIS is a valid means of assessing the teacher's load reduction instructional approaches.

\subsubsection{Load Reduction Instruction}

Load Reduction Instruction (LRI) is an instructional approach to manage students' cognitive burden and encourage guided independent learning. LRI assesses the teacher's load reduction instructional approaches through five factors. These factors reduce the difficulty of instruction during initial learning, instructional support and scaffolding, ample structured practice, appropriate provision of instructional feedback, and independent application (Martin \& Evans, 2019). Martin and Evans (2019) examined the validity and the underlying conceptual contentions of the Load Reduction Instruction Scale (LRIS). The LRIS aims at assessing the five factors included in LRI.

\subsubsection{Academic Tutoring}

The research on academic tutoring has focused on improving academic performance such as grades, GPA, and program retention. These factors are often assessed to demonstrate the effectiveness of tutoring. On average, $25 \%$ to $37 \%$ of students responded that they had received tutoring (Garcia-Perez et al., 2021). The percentage of students seeking tutoring was found to be even less in DPT programs, reported as 21.7\% (Owens et al., 2018). 
More female students participated in tutoring services compared to male students (Garcia-Perez et al., 2021). Regardless of gender, students had an overwhelmingly positive view of both peer tutoring and faculty lead tutoring (Garcia-Perez et al., 2021). Attempts to rely on web-based tutoring without a peer or faculty tutor were not well received by students, garnering more negative reactions, which may not be a reason to disregard the strategy (Eimer et al., 2020). Students experienced an improvement in grades, GPA, satisfaction, and retention following participation in tutoring services, indicating an effective strategy for the learner.

\subsection{Hypotheses and Their Correspondence to Research Design}

The purpose of this non-experimental quantitative design study was to identify Doctor of Physical Therapy (DPT) students' perception of cognitive load in an accelerated program with a blended curriculum as measured by the adapted Cognitive Load Scale (CLS) by the end of the third trimester of the first professional DPT program. This study identified physical therapist students' perception of cognitive load as measured by the adapted Cognitive Load Scale at the beginning of the fourth trimester. The data were further analyzed to determine if a difference existed between male and female physical therapist students' perception of cognitive overload.

\section{Hypotheses}

Null (Ho) Hypotheses:

Ho1. Male physical therapist students have no evidence of significantly higher levels of cognitive overload scores relative to female physical therapist students at statistically significant levels in an accelerated Doctor of Physical Therapy program with a blended curriculum among first professional DPT students at the beginning of their fourth trimester (RQ 1)

Ho2. The participation in academic tutoring services does not influence student's perceptions of cognitive overload in an accelerated Doctor of Physical Therapy program with a blended curriculum among first professional DPT students at the beginning of their fourth trimester (RQ 2)

Ho3. There is no moderating relationship between those students reporting participating in academic tutoring services and gender in an accelerated Doctor of Physical Therapy program with a blended curriculum among first professional DPT students at the beginning of their fourth trimester (RQ 3)

\section{Method}

This research study used a quantitative and non-experimental survey method through a parametric multiple linear regression analysis. A quantitative research design involves a large sample of participants and does not require a significant amount of time for data collection. In the proposed study, a quantitative research design was chosen to collect data and analyze the relationships between the dependent and independent variables to test the null hypotheses.

A parametric multiple linear regression analysis explains the relationship between the independent and dependent variables, where the responses are quantitative (Mahmoud, 2021). A parametric multiple linear regression analysis assisted in predicting the relationships between gender and perception of cognitive load, academic tutoring services as a moderator between cognitive load and gender and building prediction models in an accelerated DPT program with a blended curriculum.

Through Survey Monkey, participants completed the adapted CLS. The software tool Survey Monkey included the adapted CLS questionnaire. Survey Monkey provided a survey completion progress bar, revealing the total number of survey questionnaires completed (Jacobsen, 2017). The numerical data explored DPT students' perception of cognitive load of an accelerated first professional DPT program with a blended curriculum and measured the statistical relationships between the variables of interests using self-reports from the participants. The demographic data determined whether the participants' perception of cognitive load in an accelerated DPT program with a blended curriculum represents the target population for generalization purposes.

\subsection{Research Questions}

The following research questions guided this study:

RQ1: Will male students evidence significantly higher cognitive overload, as measured by the Adapted Cognitive Overload Scale, relative to female students in an accelerated Doctor of Physical Therapy program with a blended curriculum among first professional DPT students at the beginning of their fourth trimester?

RQ2: How does the participation in academic tutoring services influence the perception of cognitive overload, as measured by the adapted Cognitive Overload Scale, in an accelerated Doctor of Physical Therapy program with a blended curriculum among first professional DPT students at the beginning of their fourth trimester? 
RQ3: Is there a relationship between students reporting participating in academic tutoring and cognitive overload, as measured by the adapted Cognitive Overload Scale, and gender in an accelerated Doctor of Physical Therapy program with a blended curriculum among first professional DPT students at the beginning of their fourth trimester?

\subsection{Participant Population and Sample}

To determine the sample size, a margin of error (alpha) and the confidence level was established. An inverse relationship exists between the sample size and margin of error. When the sample size increases, the margin of error decreases. As the margin of error increases, the higher the likelihood that the research results are inaccurate (Story \& Tait, 2019). The margin of error establishes the deviation between the participants' opinions and the entire adult learner population's opinion. The margin of error was set at $5 \%$.

The confidence level (statistical power) reveals the confidence of the results of the study within the boundaries of the margin of errors, which was set at $95 \%$ (Charness et al., 2013). A response rate projects the number of participants completing the survey and was set at 30\% (Liu \& Jansen, 2018). In terms of statistical power, the G*power software indicated that within a multiple linear regression model with four predictors, a medium effect size effect (Cohen's $f=.15$ ) would be detected with power set at .95 and alpha set at .05 , using a sample size of 36 study participants. Thus, the projected sample of study participants provided sufficient statistical power for the research analysis.

The projected convenience sample of the study consisted of 36 male and female entry-level DPT students that had completed the third trimester of an accelerated DPT program with a blended curriculum. A convenience sample of 36 participants provided enough power in reference to the relationship between the variables and is more representative of the general population. The participants were enrolled in the fourth trimester of the residential program across all five campuses of the for-profit institution and met the inclusion criteria. The sample was consistent with the aims for this study and represented the general physical therapist students' population enrolled in an accelerated DPT program with a blended curriculum.

\subsection{Research Design}

This research study utilized the adapted CLS to measure physical therapist students' perception of cognitive load in an accelerated DPT program with blended learning at the beginning of the fourth trimester. The adapted CLS measured intrinsic and extraneous cognitive load, attendance, and frequency of academic tutoring services. The survey design was used to answer the research questions and tested the null and alternative hypotheses, regarding students' perception of cognitive load, the relationship between higher levels of cognitive overload, and differences between female and male perceptions of cognitive overload. The survey design also investigated the relationship between academic tutoring services and cognitive load perception and how receiving academic tutoring services moderated the relationship between cognitive overload and gender. Surveys allow researchers to collect data anonymously from sensitive populations, such as students (Brasel et al., 2020). A parametric multiple linear regression analysis helped predict the relationship between the dependent and independent variables with a large sample size (Mahmoud, 2021).

\subsubsection{Recruitment}

This study focused on a residential accelerated DPT program with blended learning. Prospective participants were informed through the institution's email addresses about the study, including identified issues in the study's problem statement, purposes, benefits, and potential risks. No personal identifiers were required. Utilizing the adapted CLS, the participants completed the survey through Survey Monkey indicating their perception of cognitive overload. Survey Monkey housed the data for data collection and analysis. The Survey Monkey delivered the adapted CLS to the participants chosen for this direct study across all campuses. The data collection occurred throughout the summer trimester of 2021 and was saved by male and female clusters. The participant's information was stored digitally with code numbers to protect their personal identifying information. The investigator also selected the appropriate sample of participants to represent the targeted population accurately (Martínez-Mesa et al., 2016) and obtained informed consent from the participants to ensure their understanding of the benefits and risks associated with the research activities and their wiliness to participate in the research study (Sandu \& Frunza, 2019). 


\section{Results}

\subsection{Analyses}

The software tool Survey Monkey included the adapted CLS questionnaire. The Survey Monkey provided a survey completion progress bar, revealing the total number of survey questionnaire completed (Waclawski, 2012). A URL was provided to the participants through the institution's email addresses to complete the survey. Once the participants completed the survey, the numeric and discrete data were clustered by gender. The numeric data was interpreted from the pre-populated answers obtained from the adapted CLS questionnaire survey, determined the top research questions, filtered the results by cross-tabulating sub-groups (male vs. female students), and ran an analysis on the SPSS software to find values that were statistically relevant to the research study.

\subsection{SPSS}

A parametric statistical multiple linear regression analysis was performed to predict the relationship between students' perception of cognitive overload and gender among DPT students in an accelerated program with a blended curriculum. The latest version on SPSS (26.0) was used for all statistical analysis. The data analysis plan was conducted in four phases. First, all study variables were presented using descriptive statistics, such as, means, standard deviation, and minimum/maximum values for continuous variables (Interval/Ratio level) and frequencies and percentages for categorical variables (Nominal/Ratio level).

The second phase of data analysis included bivariate testing. Specifically, a series of bivariate tests were used to determine which explanatory variables (i.e., independent and covariate variables) were related to each dependent variable at a statistically significant level $(\mathrm{p}<.05)$. Independent samples $\mathrm{t}$-test analysis examined dichotomous explanatory variables with each dependent variable. One-Way ANOVA examined categorical explanatory variables (three or more categories) with each dependent variable. Pearson's $r$ correlations analysis examined continuous explanatory variables with each dependent variable. All explanatory variables significantly related to a dependent variable in bivariate analysis included in the third phase of data analysis, multivariate analysis, for that respective dependent variable.

The third phase of data analysis was multivariate analysis. In this phase, two separate multiple linear regression (MLR) models were tested. The first MLR modeled the dependent variable as a function of the independent variable cognitive overload along with the covariate variables significantly related to this dependent variable in bivariate analysis. The second MLR modeled the dependent variable cognitive overload as a function of the independent variables Gender and Receiving Academic Tutoring Services (Yes/No) along with the covariate variables significantly related to this dependent variable in bivariate analysis. Each multiple linear regression model was assessed in terms of overall statistical significance, the significance of individual predictors, and beta values. Final hypothesis testing was based upon the findings of the multivariate analysis.

The fourth phase of data analysis involved testing for moderating effects. Specifically, an interaction term (Cognitive Overload X Receiving Academic Tutoring Services) was entered into the MLR model, examining the dependent variable to examine if receiving academic tutoring services moderated the relationship between cognitive overload and gender. If the interaction term was statistically significant in the context of the MLR model, to examine the interactive effect, mean dependent variable scores were plotted upon a graph reflecting Receiving Academic Tutoring Services (Yes/No) by low and high levels of cognitive overload. Low levels of cognitive overload will be defined as scores 1 standard deviation below the mean value cognitive overload score. High levels of cognitive overload were defined as scores 1 standard deviation above the mean value cognitive overload score.

\subsection{Analysis of the Findings}

The quantitative study examined DPT students' perception of cognitive load and determined how academic tutoring services and gender affected the participants' perception of cognitive load in an accelerated DPT program with a blended curriculum at a for-profit university in the United States. Limited research exists examining blended learning as a curricular model in physical therapy education (Gagnon et al., 2020). Educators need to consider student mental effort in working memory when learning to minimize cognitive overload (Leppink \& Duvivier, 2016). Nazari et al. (2020) found a high correlation between objective and subjective cognitive load measures. The adapted Cognitive Load Scale provided the parameters to measure student perception of cognitive load, and attendance of academic tutoring services and its frequency. In the literature review, most of the studies did not determine student perception and gender related trends (Castro-Alonso et al., 2019; Sweller, 2019; Zotova, 2015; Wei, 2017). Therefore, the adapted Cognitive load Scale included participant's gender.

Research findings indicated no relationship exists between cognitive load and gender since an independent-samples t-test analysis indicated that cognitive overload scores were not significantly related to 
gender. These findings contribute to research since studies in the literature review did not determine related trends between student perception of cognitive load and gender. The limited G*Power of 36 participants may be a limitation of these findings. The measurement tool, type of curriculum, curriculum content and student body may also serve as limiting factors.

The research on academic tutoring services has focused on improving academic performance as measured by grades, GPA, and program retention. The literature review revealed that $21.7 \%$ of DPT students seek academic tutoring services in DPT programs (Owens et al., 2018). In general, female students' participation in academic tutoring services is higher relative to male students (Garcia-Perez et al., 2021). Findings from the research study found a significant difference between the participants who reported receiving academic tutoring services and cognitive load scores. These findings are in line with previous studies, indicating that students have positive views of academic tutoring services (Garcia-Perez et al., 2021). An independent-samples $t$-test analysis indicated that lower cognitive overload scores were significantly related to attending tutoring services during at least one trimester relative to those who did not in a blended curriculum.

Analysis of the data demonstrated that attending tutoring services during at least one trimester did not moderate the relationship between gender and cognitive overload. Multiple linear regression indicated that no moderating relationship exists between those students reporting participating in academic tutoring services and gender in an accelerated Doctor of Physical Therapy program with a blended curriculum among first professional DPT students at the end of their first trimester. The moderating effect, at most nine participants, are representative of the entire male population receive academic tutoring services. Since most participants described a higher level of cognitive overload, it is difficult to compare them with the other cognitive load categories (low and medium), as indicated in the adapted Cognitive Load Scale.

The analysis found no significant relationships between the perception of cognitive overload by gender, as indicated by the analysis of an independent-samples $t$-test (Table 1). The perception of cognitive overload was related to having attended academic tutoring services for at least one trimester, specifically participants who had tutoring services had lower cognitive lover load scores as compared to those who did not receive academic tutoring services, which was also indicated by analyzing an independent-samples $t$-test (Table 1).

Table 1. Summary of the Results of Hypothesis Testing for Research Questions (RQ) with Respect to Rejecting or Accepting the Null Hypothesis

\begin{tabular}{llll}
\hline RQ & Adapted Cognitive Load Subscale & Reject Null & Accept Null \\
\hline 1 & Gender & $\mathrm{x}$ \\
2 & Academic Tutoring Services & $\mathrm{x}$ & $\mathrm{x}$ \\
3 & Gender by Academic Tutoring Services & & \\
\hline
\end{tabular}

Finally, a multiple linear regression indicated that attending academic tutoring services did not moderate the relationship between gender and the perception of cognitive overload. Cognitive overload scores were significantly related to having attended tutoring services during at least one trimester with lower cognitive overload scores among those who responded Yes relative to those who responded No. The impact of tutoring services had a small/medium effect size on reducing cognitive overload (Cohen's $\mathrm{D}=.31$ ).

Research question 1 represented the examination of how gender influences the perception of cognitive load. The null hypothesis 1 was accepted since no evidence was found that gender influences the perception of cognitive load. Table 1 shows a descriptive analysis of categorical demographic characteristics. An independent-samples t-test analysis indicated that no significant difference between cognitive overload scores and gender, $\mathrm{t}(32)=.37, \mathrm{p}=.71$.

Research question 2 represented the relationship between receiving academic tutoring services and cognitive load scores. The null hypothesis 2 was rejected. An independent-samples $t$-test analysis, indicating that cognitive overload scores were significantly lower related to having attended tutoring services during at least one trimester, $\mathrm{t}(26.09)=-3.09, \mathrm{p}<.01$ was conducted (Table 2$)$. 
Table 2. Independent Samples T-Test Analysis of Cognitive Overload Scores by Independent Variables $(n=36)$

\begin{tabular}{lllll}
\hline Variable & $\mathbf{n}$ & $\mathbf{M}(\mathbf{S D})$ & $\mathbf{t} / \mathbf{F}(\mathbf{d f})$ & $\mathbf{p}$ \\
\hline Gender & & & $.37(32)$ & .71 \\
Male & 19 & $5.80(.31)$ & & \\
Female & 15 & $5.76(.36)$ & & \\
\hline Tutoring Services Attendance During At Least One & & & $-3.09(26.09)$ & .005 \\
Trimester (1 ${ }^{\text {st }} 2^{\text {nd }}$, or $3^{\text {rd }}$ Trimester) & & & \\
Yes & 20 & $5.65(.39)$ & \\
No & 16 & $5.94(.16)$ & & \\
\hline
\end{tabular}

Those participants who responded "Yes" recorded lower cognitive overload scores $(\mathrm{M}=5.65, \mathrm{SD}=.39)$ relative to those who responded No $(\mathrm{M}=5.94, \mathrm{SD}=.16)$.

Lastly, research question 3 represented the moderating effect of academic tutoring on cognitive load. Null hypothesis 3 was accepted. Table 3 presents a multiple linear regression analysis, indicating that attending academic tutoring services during at least one trimester did not moderate the relationship between gender and cognitive overload, $B=-.12, \mathrm{SE}=.22, p=.59$.

Table 3. Multiple Linear Examining the Moderating Effect of Attending Tutoring Services During At Least One Trimester on the Relationship Between Gender and Cognitive Overload ( $\mathrm{n}=36)$

\begin{tabular}{llll}
\hline Variable & B (SE) & $\boldsymbol{\beta}$ & p \\
\hline Gender & $.01(.16)$ & .02 & .95 \\
Attended Tutoring Services & $-.10(.33)$ & -.15 & .77 \\
During At Least One Trimester (ATSDALOT) Gender X & $-.12(.22)$ & -.29 & .59 \\
ATSDALOT & & & \\
\hline
\end{tabular}

$\mathrm{F}(33)=2.19, \mathrm{p}=.11, \mathrm{R}^{2}-.18$, Adjusted $\mathrm{R}^{2}=.10$.

Since null hypotheses 1 and 3 were accepted, and null hypothesis 2 was rejected, null hypothesis 2 demonstrated significant results. In summary, research question 2 demonstrated significant results since the null hypothesis was rejected. Research questions 1 and 3 demonstrated no significant results since the null hypotheses were accepted.

\section{Discussion}

The research study offers valuable information to DPT students, educators in physical therapy education, and administrators of an accelerated DPT curriculum with a blended learning. DPT students beginning an accelerated DPT program with blended learning should be educated on the complexity and cognitive demand of blending learning. The development of organizational skills and motivational factors to minimize cognitive overload is vital for success. Those students that experience cognitive overload, experience academic performance challenges, or are identified as being at-risk for failure may benefit from academic tutoring services within the first year of the curriculum.

Physical therapy educators in an accelerated program with blended learning should be aware of student perception of cognitive overload. Instructional methods and learning materials need to be carefully designed and selected to minimize cognitive overload while maintaining academic rigor and educational expectations. Academic tutoring services may decrease student perception of cognitive overload. Minimizing cognitive load among DPT students in an accelerated DPT program with blended learning is vital for learning and academic success. The blended learning environment may lead to cognitive overload and unsatisfactory academic performance in blended learning (Castro-Alonso et al., 2019; Sweller, 2019; Wei, 2017; Zotova, 2015). Task fidelity, task complexity, and instructional support are core issues contributing to student perception of cognitive overload (Leppink \& Duvivier, 2016). A holistic model that facilitates the transition from high instructional support on low-complexity low-fidelity learning tasks to high-complexity tasks in high-fidelity environments minimizes cognitive overload and enables the transition from the classroom to the clinical setting (Zotova, 2015). 
The limitations of the research study impact the generalization of the study findings. The study included a target population of one institution, one trimester during the first year of the curriculum, a convenience sampling, the participants' apprehension to participate in the study, an adapted version of the CLS, and limited demographic characteristics. Research was conducted at one private, not-for-profit institution across five campuses. The study did not measure the number of participants per campus. It is not likely that 36 participants can represent all five campuses, limiting generalizability. The participants consisted of students in the fourth trimester only of the curriculum. The sample of convenience consisted of DPT students. The CLS is a validated tool to measure cognitive load within classroom settings with problem-based learning methods (Leppink \& Duvivier, 2016). A tool measuring tool cognitive load in an accelerated program with a blended learning has not been validated, thus, the CLS was adapted with permission to modify and create questions for the participants. The study measured two demographics, gender, and age group.

Future research recommendations include determining the DPT students' perception of cognitive overload in accelerated DPT programs with blended learning in different institutions across the United States. It is recommended that future research to determine if there is a statistical difference related to perception of cognitive load based on sexual orientation categories such as: lesbian, gay, bisexual, transgender, and queer's perception of cognitive overload is statistically different. Although the different sexual orientation categories were included in the measurement tool for this study, the participants only selected male or female.

Future studies may also examine how academic tutoring services influence students' perception of cognitive overload throughout the DPT curriculum. The research study examined how cognitive overload was influenced by attending academic tutoring services only in the fourth trimester of the curriculum. The final recommendation is to determine the perception of cognitive overload in the different course tracks, such as the non-clinical courses, neurology/pediatric, orthopedic, and medical course sequences. These recommendations for future research may be beneficial to academic institutions offering the DPT program and other health-related fields, the course designers, and the instructors. The optima goal is to determine how different independent variables influence students' perception of cognitive overload, re-design curricula to minimize cognitive load while maintaining rigor and meeting the objectives of the courses, facilitate learning and the transition from the classroom to the clinical setting, while fostering student well-being.

The purpose of this non-experimental quantitative study was to identify DPT students' perception of cognitive overload drawn on the CLT and the influence of attending academic tutoring services and gender on cognitive overload in an accelerated program with blended learning. Findings from the research indicated no significant differences mean of cognitive overload scores among male and female participants in an accelerated DPT program with a blended curriculum as measured by the adapted CLS. This is valuable information for accelerated programs to target male and female students effectively. For instance, educators may not need to design and implement different instructional methods that are gender specific. The findings of this study also contribute to the gap in the literature examining DPT students' perception of cognitive load in an accelerated program with blended learning.

Research results also suggested that academic tutoring services may decrease the DPT students' perception of cognitive overload in an accelerated program with blended learning. The study findings provide valuable information for accelerated programs with blended learning where students report high level of cognitive overload with the curriculum load. The outcomes of this study and the suggested recommendations for future research may assist DPT programs to assess and re-structure the curricula to minimize the perception of cognitive overload in blended learning while promoting active and cooperative learning for DPT students.

\section{Competing Interests Statement}

The authors declare that there are no competing or potential conflicts of interest.

\section{References}

Arco-Tirado, J. L., Fernández-Martín, F. D., \& Hervás-Torres, M. (2019). Evidence-based peer-tutoring program to improve students' performance at the university. Studies in Higher Education, 45(11), 2190-2202. https://doi.org/10.1080/03075079.2019.1597038

Brasel, K., Haider, A., \& Haukoos, J. (2020). Practical guide to survey research. JAMA Surg, 155(4), 351-352. https://doi.org/10.1001/jamasurg.2019.4401

Castro-Alonso, J. C., Wong, M., Adesope, O. O., Ayres, P., \& Paas, F. (2019). Gender imbalance in instructional dynamic versus static visualizations: A meta-analysis. Educational Psychology Review. https://doi.org/10.1007/s10648-019-09469-1 
Charness, G., Rustichini, A., \& van de Ven, J. (2013). Self-Confidence and strategic behavior. SSRN Electronic Journal. https://doi.org/10.2139/ssrn.2361183

Costley, J., \& Lange, C. (2017). The effects of lecture diversity on germane load. International Review of Research in Open and Distributed Learning, 18 (2), 27-46. https://doi.org/10.19173/irrodl.v18i2.2860

Eimer, C., Duschek, M., Jung, A. E., Zick, G., Caliebe, A., Lindner, M., Weiler, N., \& Elke, G. (2020). Video-based, student tutor- versus faculty staff-led ultrasound course for medical students: A prospective randomized study. BMC Medical Education, 20(1). https://doi.org/10.1186/s12909-020-02431-8

Gagnon, K., Young, B., Bachman, T., Longbottom, T., Severin, R., \& Walker, M. J. (2020). Doctor of physical therapy education in a hybrid learning environment: Reimagining the possibilities and navigating a "new normal." Physical Therapy. https://doi.org/10.1093/ptj/pzaa096

García-Pérez, L., García-Garnica, M., \& Olmedo-Moreno, E. M. (2021). Skills for a Working Future: How to Bring about Professional Success from the Educational Setting. Education Sciences, 11(1). https://doi.org/10.3390/educsci11010027

Jacobsen, K. (2017). Introduction to health research methods: A practical Guide (2nd edition). Sudbury, MA: Jones and Bartlett Publishers.

Jonker, H., März, V., \& Voogt, J. (2020). Curriculum flexibility in a blended curriculum. Australasian Journal of Educational Technology, 36(1), 68-84. https://doi.org/10.14742/ajet.4926

Khan, E. (2015). Cognitive overload management: Empowering simulation based medical education through mindful reality checks. BMJ Simulation and Technology Enhanced Learning, 1(1). https://doi.org/10.1136/bmjstel-2015-000044.12

Klepsch, M., Schmitz, F., \& Seufert, T. (2017). Development and validation of two instruments measuring intrinsic, extraneous, and germane cognitive load. Frontiers in Psychology, 8. https://doi.org/10.3389/fpsyg.2017.01997

Leppink, J., \& van den Heuvel, A. (2015). The evolution of cognitive load theory and its application to medical education. Perspect Med Educ, 4, 119-127. https://doi.org/10.1007/s40037-015-0192-x

Leppink, J. \& Duvivier, R. (2016). Twelve tips for medical curriculum design from a cognitive load theory perspective. Medical Teacher, 38(7), 669-674. https://doi.org/10.3109/0142159X.2015.1132829

Liu, Z., \& Jansen, B. J. (2018). Questioner or question: Predicting the response rate in social question and answering on Sina Weibo. Information Processing \& Management, 54(2), 159-174. https://doi.org/10.1016/j.ipm.2017.10.004

Mahmoud, H. F. (2021). Parametric versus semi and nonparametric regression models. International Journal of Statistics and Probability, 10(2), 90. https://doi.org/10.5539/ijsp.v10n2p90

Martin, A. J., \& Evans, P. (2019). Load reduction instruction (LRI). Advances in Cognitive Load Theory, 15-29. https://doi.org/10.4324/9780429283895-2

Martínez-Mesa, J., González-Chica, D. A., Duquia, R. P., Bonamigo, R. R., \& Bastos, J. L. (2016). Sampling: How to select participants in my research study? Anais Brasileiros De Dermatologia, 91(3), 326-330. https://doi.org/10.1590/abd1806-4841.20165254

Mayer, R. E. (2017). Using multimedia for e-learning. Journal of Computer Assisted Learning. https://doi.org/10.1111/jcal.12197

Nazari, T., van de Graaf, F. W., Dankbaar, M. E. W., Lange, J. F., van Merriënboer, J. J. G., \& Wiggers, T. (2020). One step at a time: Step by step versus continuous video-based learning to prepare medical students for performing surgical procedures. Journal of Surgical Education, 77(4), 779-787. https://doi.org/10.1016/j.jsurg.2020.02.020

Owens, S. C., Rainey, Y., Tucker, P., \& Edmunds, B. (2018). Effectiveness of a retention program to improve performance during the first semester of a doctor of physical therapy program. Journal of Health Care for the Poor and Underserved, 29(1), 430-447. https://doi.org/10.1353/hpu.2018.0029

Picardo, A. L. (2017). Implications of acceleration on student success course curriculum: A comparison of accelerated and traditional course modalities (dissertation).

Sandu, A., \& Frunza, A. (2019). Informed consent in research involving human subjects. Advances in Library and Information Science Ethics in Research Practice and Innovation, 171-191. 
https://doi.org/10.4018/978-1-5225-6310-5.ch009

Story, D. A., \& Tait, A. R. (2019). Survey research. Anesthesiology, 130, 192-202. https://doi.org/10.1097/ALN.0000000000002436

Sweller, J. (1988). Cognitive load during problem solving: Effects on learning. Cognitive Science, 12(2), 257-285. https://doi.org/10.1207/s15516709 $\operatorname{cog} 1202 \_4$

Sweller, J., Merriënboer, J. J. G. V., \& Paas, F. (2019). Cognitive architecture and instructional design: 20 years later. Educational Psychology Review, 31(2), 261-292. https://doi.org/10.1007/s10648-019-09465-5

Waclawski, E. (2012). How I use it: Survey monkey. Occupational Medicine, 62(6), 477-477. https://doi.org/10.1093/occmed/kqs075

Wei, Y. (2017). Explore the blended teaching model from the perspective of cognitive load. Advances in Social Science, Education and Humanities Research, 264. https://doi.org/10.2991/icemaess-18.2018.18

Zotova, O. (2015). Information overload as a factor of stress for students. 2nd International Multidisciplinary Scientific Conference on Social Sciences and Arts SGEM2015. https://doi.org/10.5593/SGEMSOCIAL2015/B11/S2.081

\section{Copyrights}

Copyright for this article is retained by the author(s), with first publication rights granted to the journal.

This is an open-access article distributed under the terms and conditions of the Creative Commons Attribution license (http://creativecommons.org/licenses/by/4.0/). 